\title{
微单倍型在法医遗传学中的研究进展
}

\author{
周 靖 ${ }^{1}$, 王 艳 ${ }^{1}$,徐恩萍 ${ }^{1,2}$ \\ 1. 浙江大学基础医学院病理学与病理生理学系, 浙江杭州 310058 \\ 2. 浙江大学司法鉴定中心, 浙江 杭州 310029
}

\begin{abstract}
[ 摘 要] 微单倍型是一种可以应用于法医遗传学的新型遗传标记, 具有多态性 高、突变率低、无 stutter 产物、扩增片段短的特点。微单倍型可以有效检出混合 斑, 并对混合斑贡献者定量分析; 对严重碎片化的DNA成功分型, 应用于降解检材; 作为始祖信息位点将全球大陆人群按照遗传结构进行有效划分; 提供比短串联重 复更多的信息量, 帮助鉴定复杂亲缘关系; 还可以为肿瘤身源鉴定、细胞系鉴定、 产前亲子鉴定提供新的思路, 因此具有非常广阔的应用前景。本文就微单倍型的 上述应用方向进行论述, 以期为法医学实践提供参考。
\end{abstract}

[关键词] 法医遗传学; 微单倍型; 生物学物证; 混合斑; 降解检材; 种族推断; 复杂亲 缘关系;综述

[中图分类号 ] R89;DF795.2 [ 文献标志码 ］A

\section{Research progress on application of microhaplotype in forensic genetics}

ZHOU Jing ${ }^{1}$, WANG Yan ${ }^{1}$, XU Enping ${ }^{1,2}$ (1. Department of Pathology and Pathophysiology, School of Basic Medical Sciences, Zhejiang University, Hangzhou 310058, China;

2. Forensic Science Center, Zhejiang University, Hangzhou 310029, China)

Corresponding author: XU Enping, E-mail: xep@zju.edu.cn, https://orcid.org/0000-00025567-9810

\begin{abstract}
[ Abstract ] As a novel genetic marker, microhaplotype can be applied in the field of forensic genetics. Microhaplotype has the advantages of high polymorphism, low mutation rate, no stutter products and short amplification fragments. Microhaplotype can effectively detect mixture, and quantitatively analyze the contributors of mixture. DNA with severe fragmentation can be successfully genotyped by microhaplotype. It can be used as ancestry informative marker to effectively divide the global continental population according to genetic structure. Microhaplotype system can provide more information than
\end{abstract}

\section{收稿日期:2021-06-29 接受日期:2021-10-26网络预发表日期:2021-12-21}

基金项目: 浙江省公益性技术应用研究计划(LGF21H230001,2016C33150); 浙江省自然科学基金(LY17H160025)

第一作者:周 靖,硕士研究生,主要从事法医遗传学研究;E-mail:21918038@zju.edu.cn;https ://orcid.org/0000-00024490-6600

通信作者:徐恩萍,副教授,博士生导师,主要从事病理学、法医遗传学研究;E-mail:xep@zju.edu.cn;https://orcid.org/ 0000-0002-5567-9810 
traditional short tandem repeat and help to identify complex relationships. It can provide new ideas for tumor source identification, cell line identification and prenatal paternity testing. Here we review the applications of microhaplotype, intending to provide references for forensic practice.

[ Key words ] Forensic genetics; Microhaplotype; Biological evidence; DNA mixture; Degraded DNA; Ancestry inference; Complex kinship testing; Review

[缩略语] 短串联重复(short tandem repeat,STR); 单核苷酸多态性(single nucleotide polymorphism, SNP); 插入/缺失多态性(insertion/deletion polymorphism,InDel); 聚合酶 链反应 (polymerase chain reaction, PCR); 始祖信息位点(ancestry informative marker, AIM)

在法医实践中,遗传学标记经历了从限制性 内切酶片段长度多态性到 STR 的变迁。STR 具有 片段短小、信息量大、遗传多态性高、易于检测等 优点, 目前已经成为法医DNA 分析中最常用、最重 要的遗传标记。但STR也存在一些问题, 如突变 率高、在高度降解检材中表现不佳、存在 stutter 产 物干扰等。 SNP 和 InDel 作为法医实践中起补充作 用的遗传标记, 较 STR 突变率更低, 且其扩增产物 可以很短, 但这两种遗传标记通常是二等位基因, 存在多态性低的缺点。

2012年, 美国耶鲁大学Kenneth Kidd教授研 究团队报道了一组小于 $10 \mathrm{~kb}$ 且包含 3 个及以上 SNP 的单倍型遗传标记, 并将其称为迷你单倍型 (mini-haplotype $)^{[1]}$ 。迷你单倍型在亲权鉴定及祖 先推断等方面具有一定价值, 但其分型受到检测 条件的限制,难以在法医学实验室推广。2013年, 该研究团队首次将微单倍型遗传标记引人法庭科 学领域 ${ }^{[2]}$ 。微单倍型是指片段长度在 $200 \mathrm{bp}$ 以 内, 包含两个及以上存在连锁不平衡 SNP 的等位 基因组合。微单倍型基因座内含有多个 SNP位 点, 因此属于多等位基因遗传标记, 相较于单个 SNP位点包含更丰富的遗传信息, 但突变率较 STR 低 5 6个数量级。由于微单倍型中无重复序列, 不 会产生 stutter 峰。因此, 微单倍型兼具STR 和SNP 遗传标记的优势, 在法医遗传学领域具有巨大潜 力。本文就目前微单倍型在法医遗传学中的应用 研究进行综述。

\section{1 微单倍型在混合斑中的应用}

混合斑是指两名或两名以上个体的血液、体
液或分泌液等混合形成的斑痕。混合斑在法医实 践中并不鲜见,最常见于多个个体的混合血痕以 及性犯罪案件中男性精液与女性受害者阴道液组 成的混合斑。目前应用于混合斑检测的遗传标记 仍以常染色体STR及Y-STR 为主。然而, 常染色体 STR 在不平衡混合斑中表现欠佳,当次要贡献者所 占比例低于 $1: 20$ 时,很难检测出来 ${ }^{[3]} ;$ Y Y-STR 对 于男女混合斑中的男性成分具有极高的灵敏 度 $^{[4]}$, 无法解决同性别不平衡混合斑的问题。其 次,STR 在 PCR 扩增过程中会发生复制滑脱,产生 stutter 峰, 加大了判定次要贡献者的难度 ${ }^{[5]}$ 。

SNP 和 InDel 遗传标记可以作为混合斑检验 的补充方案, 其共同特点是均不存在 stutter 峰, 但 其多为二等位基因,能提供的信息有限。近年来, 复合遗传标记作为一种新的补充方法, 可以通过 设计特异性引物使不平衡标本中的次要贡献者更 易检测出来 ${ }^{[6]}$ 。但这种方法亦有缺点, 主要是需 要使用参考DNA标本来确定次要贡献者的分型。

为了量化微单倍型基因座检出混合斑的概 率, Kidd 等 ${ }^{[7]}$ 引人了群体遗传学中有效等位基因 数 $A_{e}$ 值的概念。 $A_{e}$ 值是一个理论值,一个基因座 的期望杂合度等于 $\mathrm{A}_{\mathrm{e}}$ 个频率完全相同的等位基因 所产生的杂合度。 21 个 $\mathrm{A}_{\mathrm{e}}$ 值为 3 的微单倍型基因 座检出混合斑的概率为 $99.99956 \%{ }^{[8]}$ 。目前, 微 单倍型的 $\mathrm{A}_{\mathrm{e}}$ 值大多为 $2 \sim 5^{[8-11]}$, Voskoboinik 等 ${ }^{[12]}$ 甚至在基因组中篮选出 10 个 $A_{\mathrm{e}}$ 值为 14.7 64. 9 的 单倍型 (长度为 189 349 bp)。

Chen 等 ${ }^{[9,13]}$ 研究表明,微单倍型不仅可以对 混合斑进行定性分析,还可以对主要贡献者和次 要贡献者进行定量分析: 利用微单倍型体系, 主要 
贡献者在平衡标本和不平衡标本中均能很好地确 定 (似然率为 $6.2 \times 10^{7} \sim 2.5 \times 10^{12}$ ), 并且如果捕获到 足够的位点, 还可以识别出次要贡献者; 等位基因 深度比与贡献者混合比之间存在相关性, 这有助 于推断每个贡献者在DNA混合物中的比例; 一些 位点捕获的等位基因还可以直接分配给主要贡献 者或次要贡献者,用于个体识别。

$\mathrm{Liu}$ 等 ${ }^{[14]}$ 将 DNA 序列中的 InDel 及其下游 200 bp内的微单倍型组合定义为DIP-微单倍型。 该团队通过 SNaPShot 技术对这种新型遗传标记进 行分析,在 $1: 1$ 至 $1: 1000$ 的混合标本中均可检 出次要贡献者的分型。因此,DIP-微单倍型因其 片段短、多态性和敏感性高, 在不平衡混合斑分析 中具有良好的应用前景。

综上所述, 相比STR 在混合斑中的应用, 微单 倍型不仅无 stutter产物, 而且由于各等位基因长度 相等减少了优势扩增现象。此外, 微单倍型相比 STR 在相同的混合斑中可以得到更多来自次要贡 献者的分型 ${ }^{[15]}$, 检测到的微单倍型等位基因数可 以帮助预测混合斑中贡献者的数量 ${ }^{[16]}$ 。因此, 混 合斑检验时微单倍型可作为STR 的有效补充。

\section{2 微单倍型在降解检材中的应用}

源于各类案件现场的许多法医生物物证检材 会因高温、潮湿、微生物酶解等作用而破坏, DNA 链断裂、分子降解 ${ }^{[17]}$ 。如何从这些高度降解的检 材中获取足量的、可靠的遗传信息来进行个体识 别和亲权鉴定, 这一直是法医遗传学面临的重大 难题和挑战之一。现有试剂盒 STR 的扩增片段长 度范围大多为 100 450 bp, 而高度降解检材的 DNA 片段长度往往小于 $100 \mathrm{bp}$, 因此在进行 PCRSTR 分型时, 常发生大片段基因座分型丢失甚至分 型失败,使得鉴定工作陷人困境 ${ }^{[18]}$ 。

针对这一难题, 目前主要的解决方法是小片 段STR(miniSTR) 分型。小片段STR通过重新设计 STR 基因座的扩增引物, 使引物尽可能靠近重复 序列区域, 将扩增产物长度减小至50 200 bp ${ }^{[19] 。}$ 该方法不仅提高了降解DNA分型的成功率, 还与 现有的STR 数据库有很好的兼容性。但该方法的 局限性在于各基因座的扩增长度差异小, 限制了 体系中的位点数; 且受限于重复序列的长度, 小片 段STR扩增片段长度不能无限减小。

除小片段 STR 外, 还有一些遗传标记可以作
为补充方法应用于降解检材。线粒体DNA不易降 解 ${ }^{[20]}$, 甚至从古残迹中所取的标本也能成功分 型; SNP 和 InDel 的扩增产物可以很短, 适用于高度 降解的检材 ${ }^{[21-22]}$ 。但这三种遗传标记也存在多态 性低、信息量不足等局限性,其中线粒体DNA属于 母系遗传且具有异质性。

微单倍型的扩增片段大小取决于该位点上相 隔最远的两个SNP之间的距离。基因组中 SNP位 点众多, 其中也不乏相隔很近的位点, 这使得微单 倍型的扩增片段可以很小。Chen 等 ${ }^{[9]}$ 选取了 26 个平均跨度为 $34 \mathrm{bp}$ 的微单倍型, 有 14 个测序成 功, 累积个体识别能力达到了 99. $9999999997 \%$; van der Gaag 等 ${ }^{[23]}$ 篮选出 16 个跨度小于 $70 \mathrm{bp}$ 的微 单倍型, 随机匹配概率的数量级在 $10^{-9}$ 到 $10^{-13}$ 间; de la Puente 等 ${ }^{[2]}$ 选取了 118 个平均跨度为 $51 \mathrm{bp}$ 的 微单倍型,其中 107 个常染色体微单倍型的随机匹 配概率达到 $3.72 \times 10^{-104}$, 该团队还利用超声处理 来模拟降解检材,发现该微单倍型体系对这些检 材的分型成功率远高于传统STR。

\section{3 微单倍型在种族推断中的应用}

在法医实践中, 对现场生物检材进行种族推 断可协助调查案件。AIM 是种族推断的重要工 具,通常是指在不同群体间等位基因频率分布差 异较大的多态性位点。一组 AIM 的联合分析不仅 可以推断某特定群体的遗传成分构成, 同时也可 以推断某一个体的祖先来源。目前应用于种族推 断的 AIM 主要为 SNP 和 InDel ${ }^{[25-26]}$ 。微单倍型与 SNP 和InDel一样具有很低的突变率, 可以在人群 中稳定遗传,因此也是一种AIM。

Kidd 教授团队篮选出 65 个平均 $\mathrm{I}_{\mathrm{n}}$ 值为 0.243 的微单倍型, 这套体系可将全球大陆人群划分至 非洲、西南亚、欧洲、南亚、中亚、东亚、美洲和太平 洋地区, 并提示遗传结构与地理边界并不完全吻 合 ${ }^{[11]}$ 。Phillips 等 ${ }^{[27]}$ 则主张在一个体系中将SNP 和微单倍型结合在一起用于祖先分析, 这套方案 的位点数与先前的 SNP 体系相当, 却能更好地区 分西亚、南亚和东亚的人群。Cheung 等 ${ }^{[28]}$ 发现微 单倍型的 $\delta 、 \mathrm{~F}_{\mathrm{ST}} 、 \mathrm{~A}_{\mathrm{e}}$ 三项指标均优于二等位基因 $\mathrm{SNP}$ 和三等位基因 $\mathrm{SNP}$, 只在 $\mathrm{I}_{\mathrm{n}}$ 这一项指标中略逊 一筹, 是区分五个大陆群体表现最好的标记。 Oldoni等 ${ }^{[29]}$ 研究显示,一个个体在其自我认同的 族群中随机匹配概率高于其他族群, 因此通过计 
算个体在不同族群中的似然比即可推测该个体所 属族群。

\section{4 微单倍型在复杂亲缘关系鉴定中的应用}

在司法实践过程中,有的案件因一些关键被 鉴定人的缺失,需要对隔代、全同胞或旁系人员进 行亲缘鉴定。而目前使用的 STR 还无法为这类复 杂亲缘关系鉴定提供足够多的信息,计算得到的 似然率值往往限制在较小的范围内, 不能得出排 除或认定的结论 ${ }^{[30-31]}$ 。

目前法医 DNA 分析中研究解决复杂亲缘关 系鉴定的技术通常集中在三个方面: X-STR、 Y-STR 和 SNP。X-STR 具有性连锁特征, 通常能 够提供比常染色体 STR 更多的信息 ${ }^{[32]}$ 。因此, 在复杂亲缘关系鉴定中, X-STR 通常被用作常染 色体 STR 的重要补充。我国司法部在 2018 年发 布了 SF/Z JD0105006-2018《法医物证鉴定 X-STR 检验规范》 ${ }^{[33]}$, 详细描述了 X-STR 在一些 复杂亲缘关系鉴定中的计算方法。但 X-STR 的 应用受到被鉴定人性别的限制, 并不适用于所有 案件。Y-STR 呈单倍型遗传, 即所有 Y-STR 基因座 的基因组合在一起作为一个整体遗传给子代。 $\mathrm{Y}$ 染色体为男性所特有, 因此Y-STR 在区分同一父 系的男性个体中具有一定的应用价值。但考虑到 Y-STR 基因座向下遗传过程中有发生突变的可能 性, 在父系亲缘关系认定中存在1 2个Y-STR 基因 座分型不一致时, 需要谨慎排除父系亲缘关系。 另外, Y-STR 与X-STR 同样受到被鉴定人性别的限 制, 只能作为辅助遗传标记。SNP作为第三代遗 传标记在法医学领域受到越来越多的关注。与 STR 相比, SNP位点具有突变率低、扩增片段短等 优势。Zhang 等 ${ }^{[34]}$ 研究表明, 40个STR 与 91 个 SNP组合的效能高于单独使用 40 个 STR, 在全同 胞检测中的敏感度和特异度均达到 $100 \%$, 且具有 较强的区分二级亲属和非亲缘关系的能力。Mo 等 ${ }^{[35]}$ 构建了一个包含 472 个 SNP 的体系用于辅助 鉴定二级亲属关系, 该体系在二级亲属鉴定中的 表现优于 19 个联合 DNA 检索系统(CODIS)核心基 因座 STR。若将 472 个 SNP 和 19 个 STR 基因座结 合起来, 则可以完全区分 136 个个体中的二级亲属 和无关个体。SNP 主要的不足是其每个位点的效 能比 STR 基因座低很多,约 6.5 个SNP才能达到 1 个STR 基因座的效能。因此, 需要通过构建包含
庞大数量位点的体系来弥补这一缺点。同时, 该 研究表明单个微单倍型基因座的效能在复杂亲缘 关系鉴定中相比SNP更高。

遗传标记可以应用于亲缘关系鉴定的必要条 件是能够稳定遗传, 已有文献报道了微单倍型在 遗传过程中的稳定性 ${ }^{[23,36-37]}$ 。de la Puente 等 ${ }^{[24]}$ 通过 100 万次模拟配对分析评估了所构建的 118 位点的微单倍型体系在复杂亲缘关系鉴定中的能 力, 全同胞/无关个体与半同胞/无关个体的似然 率分布不重叠, 因此可以很好地进行区分; 而第一 代堂表兄弟与第二代堂表兄弟则需要更多的位点 才能与无关个体区分。Sun等 ${ }^{[38]}$ 采用来自 6 个家 族 53 个个体的标本构建了 38 对亲子、5 对叔侄/ 姑侄/祖孙、29对全同胞的微单倍型体系, 进行 2000 次模拟配对分析, 对 36 个多态性较好的微单 倍型位点进行评估 (其中 30 个微单倍型位点均包 含了一个多等位基因 SNP), 该体系相比 STR 能更 有效地防止将近亲关系判定为亲子关系, 可以更 好地进行亲子鉴定及全同胞鉴定。

上述研究中似然率的计算均基于血缘一致性 法。事实上,在复杂亲缘关系鉴定中还有一些特 殊的方法, 如家系重建法、状态一致性评分法、判 别函数法、第五等位基因排除法等。一个拥有庞 大数量位点的微单倍型体系可以比STR 包含更多 的信息量,期待今后将微单倍型遗传标记与上述 各种方法相结合, 从而解决在复杂亲缘关系鉴定 中难以给出明确鉴定意见的问题。

\section{5 微单倍型在法医遗传学中的其他应用}

在法医实践中还有一类疑难检材为肿瘤组 织。在患者诉医院调错组织导致其误诊,或保险公 司诉患者提供的组织不是源自本人的情况下, 就不 得不对肿瘤组织进行身源鉴定。然而, 由于许多肿 瘤 (尤其是子宫内膜癌、结直肠腺癌)存在微卫星不 稳定现象会导致传统的 STR 分型发生改变, 如 Chen 等 ${ }^{[39]}$ 发现 62.4\% 胃肠道癌患者的肿瘤组织 STR 分型发生改变, 因此突变率较低的微单倍型似 乎在肿瘤身源鉴定中具有良好的应用前景。

在科研领域, 为保证实验数据的可靠性, 投稿 人有时需要出具实验细胞的鉴定报告以确定细胞 的 “身份”。这项鉴定工作已成为法医鉴定的分 支,一般采用常规STR检测。但若这些细胞系为肿 瘤细胞, 即使未发生交叉污染, 仍然有可能随着传 
代数的增加而出现 STR 分型的改变。因此, 认定为 同一细胞系的 STR 分型匹配值一般设定在 $80 \%{ }^{[40]}$ 。对于同一来源的细胞系, SNP 分析获得的 匹配值比STR 更高, 这可能是因为细胞系的微卫星 不稳定性对STR 分型的影响更大 ${ }^{[41]}$ 。因此, 微单 倍型可能可以为细胞系鉴定提供更准确的结果。

此外, 无创性产前亲子鉴定时由于孕妇外周 血胎儿游离核酸占比很小且碎片化严重, 传统的 STR 基因分型很难获得足够的信息位点, 微单倍 型或许可应用于产前亲子鉴定 ${ }^{[42]}$ 。

\section{6 结 语}

微单倍型遗传标记被提出至今仅有短短几年 时间, 却已经在法医遗传学领域中展现出其独特 的优势。然而, 微单倍型的发展也面临一些困难 和挑战: 首先, 微单倍型的群体遗传数据目前还不 完善, 尤其缺少我国人群的相关数据; 其次, 我国 是一个多民族聚居的国家, 需要根据我国人群的 遗传结构评估相应位点对于不同应用需求的适用 性, 这部分工作还有待进一步展开; 最后, 目前微 单倍型检测的 “金标准” ——高通量测序的成本 比传统毛细管电泳高, 且在测序数据的解释上也 尚未有统一标准, 因此该方法还未能在法医学实 验室普及。综上, 微单倍型遗传标记目前尚无法 取代主流 STR 遗传标记; 但随着研究的深人, 微单 倍型遗传标记作为 STR 的一种有效补充工具, 在 法医遗传学领域更多的应用将逐步挖掘出来。

\section{利益冲突 所有作者均声明不存在利益冲突}

\section{参考文献}

[1] PAKSTIS A J, FANG R, FURTADO M R, et al. Minihaplotypes as lineage informative SNPs and ancestry inference SNPs $[J]$. Eur J Hum Genet, 2012, 20(11): 1148-1154.

[2] KIDD K K, PAKSTIS A J, SPEED W C, et al. Microhaplotype loci are a powerful new type of forensic marker[J/OL]. Forensic Sci Int-Genet Supplement Ser, 2013, 4(1): e123-e124.

[3] OLDONI F, PODINI D. Forensic molecular biomarkers for mixture analysis [J]. Forensic Sci Int-Genet, 2019, 41: 107-119.

[4] JUNG J Y, PARK J H, OH Y L, et al. Forensic genetic study of 29 Y-STRs in Korean population $[\mathrm{J}]$. Legal Med, 2016, 23: 17-20.

[5] VAN DER GAAG K J, DE LEEUW R H, HOOGEN-
BOOM J, et al. Massively parallel sequencing of short tandem repeats— population data and mixture analysis results for the PowerSeq ${ }^{\mathrm{TM}}$ system $[\mathrm{J}]$. Forensic Sci Int-Genet, 2016, 24: 86-96.

[6] OLdONi F, CASTELla V, GROSJEAN F, et al. Sensitive DIP-STR markers for the analysis of unbalanced mixtures from "touch" DNA samples[J]. Forensic Sci Int-Genet, 2017, 28: 111-117.

[7] KIDD K K, SPEED W C. Criteria for selecting microhaplotypes: mixture detection and deconvolution $[\mathrm{J}]$. Invest Genet, 2015, 6(1): 1.

[8] KIDD K K, SPEED W C, PAKSTIS A J, et al. Evaluating 130 microhaplotypes across a global set of 83 populations $[\mathrm{J}]$. Forensic Sci Int-Genet, 2017, 29: 29-37.

[9] CHEN P, YIN C, LI Z, et al. Evaluation of the Microhaplotypes panel for DNA mixture analyses $[\mathrm{J}]$. Forensic Sci Int-Genet, 2018, 35: 149-155.

[10] TURCHI C, MELCHIONDA F, PESARESI M, et al. Evaluation of a microhaplotypes panel for forensic genetics using massive parallel sequencing technology $[\mathrm{J}]$. Forensic Sci Int-Genet, 2019, 41: 120-127.

[11] BUlbul O, PAKSTIS A J, SOUNDARARAJAN U, et al. Ancestry inference of 96 population samples using microhaplotypes[J]. Int J Legal Med, 2018, 132(3): 703-711.

[12] VOSKOBOINIK L, MOTRO U, DARVASI A. Facilitating complex DNA mixture interpretation by sequencing highly polymorphic haplotypes $[\mathrm{J}]$. Forensic Sci Int-Genet, 2018, 35: 136-140.

[13] CHEN P, DENG C, LI Z, et al. A microhaplotypes panel for massively parallel sequencing analysis of DNA mixtures[J]. Forensic Sci Int-Genet, 2019, 40: 140-149.

[14] LIU J, HAO T, CHENG X, et al. DIP-microhaplotypes: new markers for detection of unbalanced DNA mixtures[J]. Int J Legal Med, 2021, 135(1): 13-21.

[15] PANG J B, RAO M, CHEN Q F, et al. A 124-plex microhaplotype panel based on next-generation sequencing developed for forensic applications $[\mathrm{J}]$. Sci Rep, 2020, 10(1): 1945.

[16] BENNETT L, OLDONI F, LONG K, et al. Mixture deconvolution by massively parallel sequencing of microhaplotypes[J]. Int J Legal Med, 2019, 133(3): 719-729.

[17] ALAEDDINI R, WALSH S J, ABBAS A. Forensic implications of genetic analyses from degraded DNAa review $[J]$. Forensic Sci Int-Genet, 2010, 4(3): 148-157.

[18] SIRIBOONPIPUTTANA T, RINTHACHAI T, SHOTIVARANON J, et al. Forensic genetic analysis of bone remain samples[J]. Forensic Sci Int, 2018, 284: 
167-175

[19] BUTLER J M, SHEN Y, MCCORD B R. The development of reduced size STR amplicons as tools for analysis of degraded DNA $[J]$. J Forensic Sci, 2003, 48(5): 2003043.

[20] WAI K T, GUNN P, BARASH M. Development of the MitoQ assay as a real-time quantification of mitochondrial DNA in degraded samples[J]. Int J Legal Med, 2019, 133(2): 411-417.

[21] MACHIDA M, TAKI T, KIBAYASHI K. Screening for single nucleotide polymorphisms in highly degraded DNA by using the amplified fragment length polymorphism technique $[J]$. Forensic Sci Int-Genet, 2017, 31: 5-11.

[22] HUANG Y, LIU C, XIAO C, et al. Development of a new 32-plex InDels panel for forensic purpose $[\mathrm{J}]$. Forensic Sci Int-Genet, 2020, 44: 102171.

[23] VAN DER GAAG K J, DE LEEUW R H, LAROS J F J, et al. Short hypervariable microhaplotypes: a novel set of very short high discriminating power loci without stutter artefacts $[J]$. Forensic Sci Int-Genet, 2018, 35: $169-175$.

[24] DE LA PUENTE M, PHILLIPS C, XAVIER C, et al. Building a custom large-scale panel of novel microhaplotypes for forensic identification using MiSeq and Ion S5 massively parallel sequencing systems $[\mathrm{J}]$. Forensic Sci Int-Genet, 2020, 45: 102213.

[25] PHILLIPS C, PARSON W, LUNDSBERG B, et al. Building a forensic ancestry panel from the ground up: the EUROFORGEN Global AIM-SNP set[J]. Forensic Sci Int-Genet, 2014, 11: 13-25.

[26] ZAUMSEGEL D, ROTHSCHILD M A, SCHNEIDER P M. A 21 marker insertion deletion polymorphism panel to study biogeographic ancestry[J]. Forensic Sci IntGenet, 2013, 7(2): 305-312.

[27] PHILLIPS C, MCNEVIN D, KIDD K K, et al. MAPlex — a massively parallel sequencing ancestry analysis multiplex for Asia-Pacific populations $[\mathrm{J}]$. Forensic Sci Int-Genet, 2019, 42: 213-226.

[28] CHEUNG E Y Y, PHILLIPS C, EDUARDOFF M, et al. Performance of ancestry-informative SNP and microhaplotype markers $[J]$. Forensic Sci IntGenet, 2019, 43: 102141.

[29] OLDONI F, HART R, LONG K, et al. Microhaplotypes for ancestry prediction $[\mathrm{J} / \mathrm{OL}]$. Forensic Sci IntGenet Supplement Ser, 2017, 6: e513-e515.

[30］易少华, 刘宇轩, 胡清清, 等. 采用家系基因型重建 法鉴定半同胞关系 1例 $[\mathrm{J}]$. 中国法医学杂志, 2015, 30(2): 184-187.

YI Shaohua, LIU Yuxuan, HU Qingqing, et al. A case of half sib relationship was identified by family genotype reconstruction $[\mathrm{J}]$. Chinese Journal of Forensic Medicine, 2015, 30(2): 184-187. (in Chinese)
[31］屈 宁, 梁 影, 杨冬桂, 等. 家系基因型重建法鉴 定半同胞关系 1例分析 [J]. 中国法医学杂志, 2019, 34(4): 404-406, 403.

QU Ning, LIANG Hao, YANG Donggui, et al. Identification of half sib relationship by family genotype reconstruction: a case analysis $[\mathrm{J}]$. Chinese Journal of Forensic Medicine, 2019, 34(4): 404-406, 403. (in Chinese)

[32] PINTO N, GUSMÃO L, AMORIM A. X-chromosome markers in kinship testing: a generalisation of the IBD approach identifying situations where their contribution is crucial $[J]$. Forensic Sci Int-Genet, 2011, 5(1): 27-32.

［33］中华人民共和国司法部司法鉴定管理局．SF/Z JD01 05006－2018法医物证鉴定X-STR 检验规范 [S]. 2018. Bureau of Judicial Forensic Administration, Ministry of Justice of the People's Republic of China. SF/Z JD0105006 - 2018. X-STR test specification for forensic physical evidence identification[S]. 2018. (in Chinese)

[34] ZHANG Q, ZHOU Z, WANG L, et al. Pairwise kinship testing with a combination of STR and SNP loci[J]. Forensic Sci Int-Genet, 2020, 46: 102265.

[35 ] MO S K, REN Z L, YANG Y R, et al. A 472-SNP panel for pairwise kinship testing of second-degree relatives[J]. Forensic Sci Int-Genet, 2018, 34: 178-185.

[36] ZHU J, CHEN P, QU S, et al. Evaluation of the microhaplotype markers in kinship analysis $[\mathrm{J}]$. Electrophoresis, 2019, 40(7): 1091-1095.

[37] ZHU J, LV M, ZHOU N, et al. Genotyping polymorphic microhaplotype markers through the Illumina ${ }^{\circledR}$ MiSeq platform for forensics $[J]$. Forensic Sci Int-Genet, 2019, 39: 1-7.

[38 ] SUN S, LIU Y, LI J, et al. Development and application of a nonbinary SNP-based microhaplotype panel for paternity testing involving close relatives $[\mathrm{J}]$. Forensic Sci Int-Genet, 2020, 46: 102255.

[39] CHEN A, ZHANG S, LI J, et al. Detecting genetic hypermutability of gastrointestinal tumor by using a forensic STR kit $[J]$. Front Med, 2020, 14(1): 101-111.

[40] CAPES-DAVIS A, REID Y A, KLINE M C, et al. Match criteria for human cell line authentication: where do we draw the line? [J]. Int J Cancer, 2013, 132(11): 2510-2519.

[41] YU M, SELVARAJ S K, LIANG-CHU M M Y, et al. A resource for cell line authentication, annotation and quality control[J]. Nature, 2015, 520(7547): 307-311.

[42] QU N, XIE Y, LI H, et al. Noninvasive prenatal paternity testing using targeted massively parallel sequencing $[\mathrm{J}]$. Transfusion, 2018, 58(7): 1792-1799.

[本文编辑 沈 敏 余 方] 